\title{
High-z nebulae: Ionization by stars or by an obscured QSO?
}

\author{
L. Binette ${ }^{1}$, B. Groves ${ }^{2}$, M. Villar-Martín ${ }^{3}$, R. A. E. Fosbury ${ }^{4}$, and D. J. Axon ${ }^{5}$ \\ 1 Instituto de Astronomía, UNAM, Ap. 70-264, 04510 DF, México \\ e-mail: binette@astroscu.unam.mx \\ 2 Mt. Stromlo Observatory, Cotter Rd., Weston Creek, ACT 2611, USA \\ e-mail: bgroves@mso.anu.edu.au \\ 3 Dept. of Natural Sciences, Univ. of Hertfordshire, College Lane, Hatfield, Herts AL10 9AB, UK \\ 4 ST-ECF, Karl Schwarzschild Str. 2, 85748 Garching-bei-München, Germany \\ e-mail: rfosbury@eso.org \\ 5 Dept. of Physics, Rochester Institute of Technology, 85 Lomb Memorial Dr., Rochester, NY 14623-5603, USA
}

Received 19 November 2002 / Accepted 8 May 2003

\begin{abstract}
The gravitational arc observed by Holden et al. at $z=3.356$ reveals strong emission lines of N IV] $\lambda 1485$, C IV $\lambda \lambda 1549$, O III] $\lambda 1665$ but no $\mathrm{N}$ V $\lambda 1240$. The lines were modelled assuming photoionization by a thermal distribution hotter than $60000 \mathrm{~K}$. This paper considers an alternative ionizing source consisting of a partially absorbed powerlaw. We compare the line ratios from a low metallicity nebula $\left(Z_{\text {total }}=0.05 Z_{\odot}\right)$ photoionized by such filtered continuum with those produced by a zero-metallicity star of $T_{\text {eff }}=80000 \mathrm{~K}$. We find that the latter generally produces stronger emission lines of $\mathrm{C}, \mathrm{N}$ and $\mathrm{O}$ than the absorbed powerlaw, even when their respective $\mathrm{He} \mathrm{I} / \mathrm{H} \beta$ ratio is matched. The absorbed powerlaw generates $\mathrm{NV} \lambda 1240$, O VI $\lambda 1035$ and [ $\mathrm{Ne} \mathrm{V}] \lambda 3426$ nebular lines weaker than the canonical direct powerlaw, although at a much higher level than the $80000 \mathrm{~K}$ stellar atmosphere. As a result of the large partially ionized zone created by the absorbed powerlaw, the optical [O I] $\lambda 6300$ and [S II] $\lambda 6731$ lines and the $\mathrm{C} \mathrm{II}] \lambda 2326$ and $\mathrm{Mg}$ II $\lambda \lambda 2800$ lines in the $\mathrm{UV}$ are much stronger than in the stellar case. These constitute the best discriminant for differentiating the predictions of the absorbed case from those of the stellar model.
\end{abstract}

Key words. line: formation - quasars: emission lines - galaxies: formation - cosmology: early Universe

\section{Introduction}

During a study of the cluster RX J0848+4456, Holden et al. (2001; hereafter HN01) reported the discovery of a gravitational arc (their components labelled $\mathrm{A}$ and $\mathrm{B}$ ) which showed an unusual narrow emission line spectrum. Their spectrum revealed relatively strong lines of N IV] $\lambda 1485$, C IV $\lambda \lambda 1549$, O III] $\lambda 1665$ but a weaker He II $\lambda 1640$ line and no detection of $\mathrm{N} v \lambda 1240$, a line which would have been expected to be present in the case of photoionization by a powerlaw. The authors indicated that the observation of the above lines plus the perceived weakness of S IV] $\lambda 1406$ and [N III] $\lambda 1749$ favor a very high ionization parameter. They conclude that the ionizing source is softer than a typical AGN (Active Galactic Nucleus) but harder than a blackbody of $60000 \mathrm{~K}$. Is a thermal distribution, presumably from stars, the only possibility?

In the current work, we consider the possibility that a modified AGN continuum might account for the main features of the Lynx arc line spectrum. More specifically, we compare the emission line spectrum resulting from photoionization by an absorbed (or filtered) AGN continuum with that resulting from a hot stellar distribution. The parameters which define the

Send offprint requests to: B; Groves, e-mail: bgroves@mso.anu.edu.au two ionizing continua are such that they result in a comparable $\mathrm{He}$ II/ $\mathrm{H} \beta$ emission line ratio. While we do not argue here that the Lynx arc spectrum necessarily requires ionization by an AGN, we believe it is important to show that such an explanation has a degree of diagnostic plausibility. Our aim is to point out observational properties that can be used as effective discriminants in such a case.

We first define the two main ionizing spectral energy distributions (ISED) being compared as well as the nebular parameters and then proceed to compare their respective emission line ratios with the aim of finding which lines can be used as discriminants. Since HN01 have not yet published a table of the observed line ratios or fluxes but rather sketched their main results, our study will be based on a differential comparison of the line ratios produced by different theoretical energy distributions which qualitatively agree with the information available in HN01.

\section{Photoionization calculations}

\subsection{The stellar energy distribution}

As thermal distribution, we used a stellar ISED of $T_{\text {eff }}=$ $80000 \mathrm{~K}$ derived from the work of Schaerer (2002), which in terms of the proportion of $\mathrm{He}^{+}$ionizing photons is equivalent 


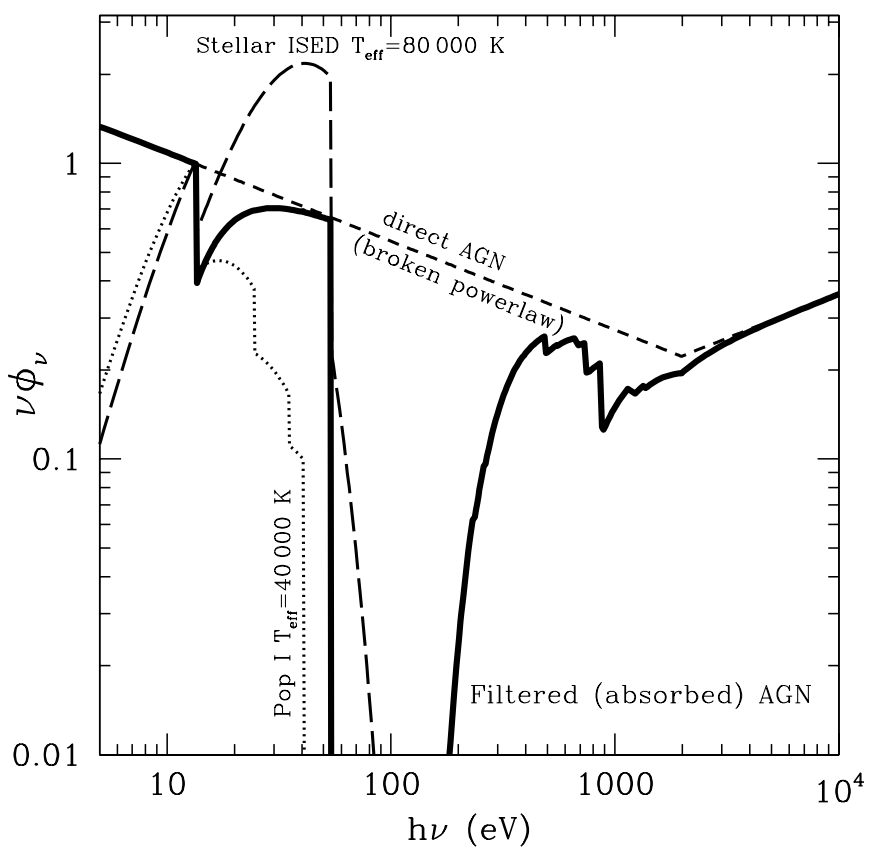

Fig. 1. Spectral energy distributions in $v \varphi_{v}$ (in arbitrary units) of the four input ionizing distributions used in the photoionization calculations presented in Table 1. These are: the direct AGN broken powerlaw (short-dashed line), the emerging filtered distribution (thick line), the zero-metallicity $80000 \mathrm{~K}$ stellar atmosphere (long-dashed line) and the $40000 \mathrm{~K}$ POP I stellar atmosphere (dotted line). The scale is arbitrary. The filtered distribution still contains $80 \%$ of the number of ionizing photons originally present in the direct broken powerlaw (Sect. 2.2).

to a blackbody of $67200 \mathrm{~K}$ (a temperature in accord with the suggestion of HN01). Although the atmospheres studied by Schaerer correspond to zero-metallicity zero-age stars, they are preferable over that of simple black bodies since they take into account the effect of $\mathrm{He}^{+}$opacity. We computed a stellar ISED that reproduced the ionizing photon luminosities $Q(\mathrm{H}), Q\left(\mathrm{He}^{0}\right)$ and $Q\left(\mathrm{He}^{+}\right)$of the model $\log T_{\text {eff }}=4.900$ found in the Table 3 of Schaerer (2002). We used the technique of Shields \& Searle (1978) to derive the monochromatic temperatures at the edge boundaries $T_{\mathrm{H}^{0}}^{+}, T_{\mathrm{He}^{+}}^{-}$and $T_{\mathrm{He}^{+}}^{+}$, and then interpolate linearly in $\log T_{\nu}$ for all the wavelengths used in the code MAPPINGS Ic. We equated $T_{\mathrm{H}^{0}}^{-}$to $T_{\text {eff }}$ and neglected the very small $\mathrm{He}^{0}$ edge present in these atmospheres. The resulting stellar energy distribution is plotted in Fig. 1 (long-dashed line). This simplified representation of a stellar atmosphere provides enough accuracy to derive the essential properties of the nebular emission lines.

\subsection{The alternative: A partly absorbed powerlaw}

The new and alternative excitation mechanism studied in this paper consists of photoionization by a partly absorbed AGN powerlaw. Dense layers of gas near the central engine and hidden from our view would be responsible for altering significantly the initial powerlaw. As explained below, the misalignment of the emerging ionizing cone relative to our line-of-sight would explain why this putative screen is hidden from our view due to the existence of a dusty and opaque torus around the black hole. The emission lines observed would originate from low density extranuclear gas (hereafter ENLR) photoinized by the resulting filtered powerlaw.

The detailed scenario is the following. We assume that a powerful QSO has been born within an opaque dense shroud of gas and dust. As the central engine's ionizing radiation eats away at the surrounding gas along two preferred (and opposite) directions $^{1}$ (the future ionization cones), it ionizes the gas and destroys its dust because of the very high value of the local ionization parameter. A strong bipolar radial wind might also be generated in the process. As the UV radiation begins to emerge from the shroud (along the direction of the ionizing cones), the radiation that initially leaks out presents a modified energy distribution with respect to the intrinsic powerlaw because the gas debris within the cones would preferentially absorb photons in the 54-80 eV range. A significant misalignment of the cone axis relative to our line-of-sight and a high scale height torus (Wada \& Norman 2002) are postulated, which means that all the lines and any scattered continuum emitted by this inner filter would be blocked from our view. The consequences of this new picture are discussed in Sect. 4.

Specifically, we will assume that the filtering gas layers absorb radiation at the very high ionization parameter $U_{\text {fil }}=2$, is very dense, $n_{\mathrm{H}}^{\mathrm{fil}} \sim 10^{11} \mathrm{~cm}^{-3}$, and has the same total metallicity as the (visible) ENLR (i.e. 5\% solar). The values of these parameters are not critical since many combinations of densities and ionization parameters can generate similar absorbed continua. The critical parameter is rather the $\mathrm{He}^{+}$opacity within the $54-80 \mathrm{eV}$ range. We have set the latter to be such that the narrow line nebular $\mathrm{He} \mathrm{I} / \mathrm{H} \beta$ ratio calculated using the $a b$ sorbed AGN continuum as input (in photoionization calculations of the ENLR) becomes comparable to that produced by the stellar ISED discussed above. With $U_{\text {fil }}=2$, this occurs when the filtering layer absorbs $\simeq 20 \%$ of $Q_{\mathrm{H}}$, the ionizing photon luminosity (by photon number).

At such a high value of $U_{\text {fil }}$, we expect the internal dust to have been destroyed. Hence the filtering component will be assumed dust-free. Within the ENLR, on the other hand, a large fraction of the dust should survive since it has $U_{\text {neb }}=0.1$, as indicated in Sect. 2.3 below. We associate the Lynx arc emission lines observed by HN01 to this external low density ENLR component. The intrinsic energy distribution assumed for the central engine (before the filter) is a broken powerlaw, (the same as in Binette et al. 1997), that is an index $\alpha_{\mathrm{UV}}=-1.3$ $\left(\varphi_{v} \propto v^{\alpha}\right)$ up to $2 \mathrm{keV}$ where the index abruptly changes to $\alpha_{\mathrm{X}}=-0.7$ up to $10^{5} \mathrm{eV}$ (where it is truncated). It is plotted in Fig. 1 (short-dashed line). Using the above parameters, the total column density of the filter turns out to be $N_{\mathrm{H}}=2.1 \times 10^{23} \mathrm{~cm}^{-2}$ and the ISED emerging from the filter and later reprocessed by the ENLR nebula is plotted in Fig. 1 (thick line).

\subsection{The gas phase abundances and dust}

In order to reveal the main differences between nebulae photoionized by an absorbed powerlaw continuum and a

${ }^{1}$ Because the gas is likely to be geometrically stratified in a thick disk-like structure rather than in a spherical shroud of gas. 
$\sim 80000 \mathrm{~K}$ stellar atmosphere, it is imperative to use the same nebular parameters: dust content, gas metallicity, gas density and ionization parameter. For the latter, we simply adopt the value favored by HN01 of $U_{\text {neb }}=0.1$ and for the total (dust + gas) nebular abundances $Z_{\text {total }}$, we adopt their suggested metallicity of $5 \%$ solar (i.e. with respect to the solar abundances of Anders \& Grevesse 1989). For helium, we assumed the primordial value of $\mathrm{He} / \mathrm{H}=0.075$. We assumed a gas density of $n_{\mathrm{H}}^{\text {neb }}=10^{3} \mathrm{~cm}^{-3}$ and a simple slab geometry.

Our models include internal dust, at a level of $2.5 \%$ of that of the solar neighborhood value, which is half of the expected value if the dust content scaled linearly with $Z_{\text {total }}$. The other missing half is assumed to have been destroyed. In effect, as a result of the very high $U_{\text {neb }}=0.1$ considered, it is unavoidable that some dust should be destroyed (Maiolino et al. 2001a,b; Lazzati \& Perna 2003). A precise calculation of the surviving dust fraction is not readily available and lies beyond the scope of this paper. To define the problem precisely, we depleted the gas phase abundances (on which depend the line brightnesses) assuming that half of the dust has been returned to the gas phase, which resulted in the following gas phase abundances: $(\mathrm{C}, \mathrm{N}, \mathrm{O}, \mathrm{Ne}, \mathrm{Mg}, \mathrm{Si}, \mathrm{S}, \mathrm{Ar}, \mathrm{Ca}$, $\mathrm{Fe})=[12,5.0,35,6.2,1.1,0.92,0.66,0.18,0.06,1.2] \times 10^{-6}$ relative to $\mathrm{H}$ (by number). This is equivalent to $Z_{\mathrm{gas}}=0.038$. The comparison between a stellar and a modified AGN continuum could have been carried out without dust but it would have been inconsistent with the general scenario of enshrouded QSOs discussed in Sect. 4. In any event, the amount of dust present in the calculations is very small given the very low metallicity of the gas. We used the code MAPPINGS Ic in our calculations (Ferruit et al. 1997). The implementation of internal dust has been described in Binette et al. (1993).

\section{Model results and discussion}

The photoionization calculations using the the $80000 \mathrm{~K}$ stellar distribution and the absorbed (broken) powerlaw distribution are presented in Cols. 3 and 4 of Table 1, respectively. For comparison purposes, we present in Cols. 5 and 7, respectively, photoionization calculations using the direct (broken) powerlaw distribution and a $40000 \mathrm{~K}$ POP I stellar distribution (Hummer \& Mihalas 1970). These ISED are plotted in Fig. 1. Note that the absorbed continuum presents a very large $\mathrm{He}^{+}$ absorption trough between $54.4 \mathrm{eV}$ and $300 \mathrm{eV}$, much deeper than that caused by $\mathrm{H}^{0}$ between 13.6 and $\sim 30 \mathrm{eV}$. This deeper trough obviously accounts for the somewhat weaker He II lines (Col. 4) of the absorbed case as compared to the direct powerlaw (Col. 5). Because the diffuse field radiated by the filtering layers is to a first order isotropic, its contibution to the ISED is negligible at the far-out position of the ENLR.

\subsection{Comparison of the nebular structure}

The very low gas metallicity is the basic explanation for the high nebular temperatures of models. This favors generating unusually strong collisionally excited UV lines which are characterized by larger excitation energies than optical lines. On the other hand, a comparison of the temperatures within the $\mathrm{C}^{+3}$ region in Table $1,\left\langle T\left(\mathrm{C}^{+3}\right)\right\rangle$, which governs the strength of C IV $\lambda \lambda 1549$, shows that similar temperature values are attained with the absorbed and direct ISED as with the $80000 \mathrm{~K}$ star. The main reason why the latter ISED is so remarkably efficient in generating strong metal UV lines is that the bulk of its ionizing photons lie within the narrow energy band $20-54.4 \mathrm{eV}$, which is crucial in maintaining a relatively large fraction of ions in the triply ionized stage (a condition facilitating the reproduction of the strong C IV and N IV] lines of the Lynx arc). This can be shown by comparing the mean ionized fractions of $\mathrm{C}$ averaged over the depth of each nebular model, $\left\langle\mathrm{C}^{+2} / \mathrm{C}\right\rangle$, $\left\langle\mathrm{C}^{+3} / \mathrm{C}\right\rangle$ and $\left\langle\mathrm{C}^{+4} / \mathrm{C}\right\rangle$, as listed in Table 1. For instance, for both the direct and absorbed powerlaw, the nebular ionization structure encompasses many different ionization stages of $\mathrm{C}$, which effectively reduces the global importance of the $\mathrm{C}^{+3}$ zone while in the case of the $80000 \mathrm{~K}$ ISED as much as $63 \%$ of $\mathrm{C}$ is triply ionized. Although the concentration of ionizing photons in the $20-54.4 \mathrm{eV}$ band of the filtered AGN ISED is comparable to that occurring in the $80000 \mathrm{~K}$ stellar distribution, the harder photons $\gtrsim 300 \mathrm{eV}$, which remain present in the absorbed ISED, generate high ionization zones, where $\mathrm{C}^{+4}$ and $\mathrm{C}^{+5}$ are present, as well as a large partially ionized zone (hereafter PIZ) with $\mathrm{C}^{+}$, all of which prevent the $\left\langle\mathrm{C}^{+3} / \mathrm{C}\right\rangle$ fraction from becoming any larger than in the direct powerlaw case. The PIZ, apart from contributing to the recombination lines, is responsible for the strong emission of the low excitation lines (Sect. 3.3).

Interestingly, if we compare the mean ionizing photoelectron energy $h\left\langle v-v_{0}\right\rangle$ of photons between 1 and 4 Ry for the various ISED in Table 1, one finds that the filtered powerlaw occupies an intermediate position between the $80000 \mathrm{~K}$ stellar distribution and the direct powerlaw. In conclusion, the gas temperature and the mean photoelectron energy, although relevant, are not the key factors driving the differences between the three hard/hot ISEDs presented in Table 1 (Cols. 3-5) but rather their respective efficiency in creating a proportionally large $\mathrm{C}^{+3}$ zone.

\subsection{Line ratio similarities}

Inspection of Table 1 shows that the absorbed powerlaw and the $80000 \mathrm{~K}$ atmosphere produce line ratios which are often comparable. For instance, comparison of Cols. 3 and 4 shows that although the CIV is twice as weak in the case of the absorbed ISED, the other strong lines like C III], N III], N IV], He II and O III] $\lambda 1665$ in the UV and [Ne III] and [O III] $\lambda 5007$ in the optical, differ by less than $50 \%$ between the two models. We ran a model with the gas metallicity three times higher $\left(Z_{\text {gas }}=3 \times 0.038 Z_{\odot}\right.$, see Col. 6$)$ in the case of the absorbed continuum (with all the other parameters the same) to determine whether the C IV line would increase to the level seen with the stellar distribution. Although collisionally excited lines have on average doubled in intensity, C IV $\lambda \lambda 1549$ still remains somewhat weaker than in the stellar case. Once a clearer picture emerges about the Lynx arc line ratios, it will be possible to vary the ionization parameter of any of the models in order 
Table 1. Line ratios from photoionization calculations ${ }^{a, b}$.

\begin{tabular}{|c|c|c|c|c|c|c|}
\hline $\begin{array}{l}\text { Line } \\
\text { ID }\end{array}$ & $\begin{array}{l}\lambda \\
(\AA) \\
Z_{\text {gas }} / Z_{\odot}: \\
(2)\end{array}$ & $\begin{array}{c}\text { Hot star } \\
80000 \mathrm{~K} \\
0.038 \\
\text { (3) }\end{array}$ & $\begin{array}{c}\text { AGN } \\
\text { Absorbed }^{c} \\
0.038 \\
(4)\end{array}$ & $\begin{array}{c}\text { AGN } \\
\text { Direct } \\
0.038 \\
(5)\end{array}$ & $\begin{array}{c}\text { AGN } \\
\text { Absorbed }^{c} \\
3 \times 0.038^{d} \\
\text { (6) }\end{array}$ & $\begin{array}{c}\text { Pop I } \\
40000 \mathrm{~K} \\
0.038 \\
(7)\end{array}$ \\
\hline $\mathrm{H} \beta$ & 4861 & 1.00 & 1.00 & 1.00 & 1.00 & 1.00 \\
\hline He II & 1640 & 0.095 & 0.13 & 1.24 & 0.14 & $<0.001$ \\
\hline He II & 4686 & 0.011 & 0.015 & 0.14 & 0.018 & $<0.001$ \\
\hline C II] & 2326 & 0.003 & 0.034 & 0.026 & 0.098 & 0.008 \\
\hline C III] & 1909 & 0.96 & 0.785 & 0.47 & 1.44 & 1.47 \\
\hline CIV & 1549 & 3.28 & 1.60 & 0.82 & 2.77 & 0.002 \\
\hline N III] & 1749 & 0.040 & 0.055 & 0.047 & 0.10 & 0.172 \\
\hline N IV] & 1485 & 0.32 & 0.25 & 0.17 & 0.40 & $<0.001$ \\
\hline $\mathrm{Nv}$ & 1240 & $<0.001$ & 0.098 & 0.20 & 0.17 & $<0.001$ \\
\hline [O I] & 6300 & 0.001 & 0.29 & 0.24 & 0.75 & $<0.001$ \\
\hline [O II] & 3727 & 0.02 & 0.09 & 0.078 & 0.27 & 0.08 \\
\hline [O III] & 5007 & 6.07 & 4.68 & 2.98 & 11.6 & 4.72 \\
\hline O III] & 1665 & 0.79 & 0.95 & 0.58 & 1.55 & 0.36 \\
\hline O VI & 1035 & $<0.001$ & 0.16 & 1.41 & 0.26 & $<0.001$ \\
\hline [S II] & 6731 & 0.002 & 0.25 & 0.20 & 0.63 & $<0.001$ \\
\hline S IV] & 1406 & 0.015 & 0.022 & 0.012 & 0.028 & 0.006 \\
\hline Mg II & 2800 & 0.016 & 0.11 & 0.084 & 0.32 & 0.013 \\
\hline Si III] & 1887 & 0.0015 & 0.0023 & 0.0022 & 0.0051 & 0.03 \\
\hline Si IV & 1397 & 0.018 & 0.022 & 0.015 & 0.043 & 0.135 \\
\hline [Ne III] & 3869 & 0.65 & 0.51 & 0.29 & 1.21 & 0.39 \\
\hline$[\mathrm{Ne} \mathrm{V}]$ & 3426 & $<0.001$ & 0.043 & 0.134 & 0.088 & $<0.001$ \\
\hline$\left\langle\mathrm{T}\left(\mathrm{H}^{+}\right)\right\rangle$ & $(\mathrm{K})$ & 21900 & 25700 & 33000 & 21200 & 18100 \\
\hline$\left\langle\mathrm{T}\left(\mathrm{C}^{+3}\right)\right\rangle$ & $(\mathrm{K})$ & 22300 & 23700 & 24300 & 19900 & 18200 \\
\hline$\left\langle\mathrm{C}^{+2} / \mathrm{C}\right\rangle$ & - & 0.363 & 0.287 & 0.192 & 0.286 & 0.988 \\
\hline$\left\langle\mathrm{C}^{+3} / \mathrm{C}\right\rangle$ & - & 0.628 & 0.207 & 0.099 & 0.223 & - \\
\hline$\left\langle\mathrm{C}^{+4} / \mathrm{C}\right\rangle$ & - & 0.005 & 0.242 & 0.273 & 0.246 & - \\
\hline$h\left\langle v-v_{0}\right\rangle_{v \geq v_{0}}^{v<4 v_{0}}$ & $(\mathrm{eV})$ & 15.2 & 12.6 & 10.4 & 12.6 & 6.4 \\
\hline
\end{tabular}

a All line ratios are expressed relative to $\mathrm{H} \beta$.

${ }^{b}$ All models assume $U_{\text {neb }}=0.1$, a density $n_{\mathrm{H}}^{\text {neb }}=10^{3} \mathrm{~cm}^{-3}$ (see Sect. 2.3) and an ionization-bounded slab geometry with the ionized fraction of $\mathrm{H}$ not exceeding $1 \%$ at the back of the slab.

${ }^{c}$ Using the filtered AGN ionizing energy distribution plotted in Fig. 1 (thick solid line).

${ }^{d}$ Model with three times higher gas phase metallicity but same dust content.

to fine-tune the fitted line ratios, a task which lies beyond the scope of the current paper.

The $40000 \mathrm{~K}$ POP I distribution is only shown for comparison (Col. 7). Because this ISED falls off abruptly at energies not far above the Rydberg limit, it cannot account for the high excitation UV lines observed in the Lynx arc nebula.

\subsection{Marked differences in line ratios}

Having shown that the strongest UV lines detected in the Lynx arc nebula (N IV], C IV, [O III] and He II) can be accounted for, in principle, by a filtered AGN continuum, we now review which differences stand up between an absorbed continuum and the $80000 \mathrm{~K}$ stellar ISED. The aim is to provide a clearcut method to distinguish between these two continua in order that future detailed observations of the Lynx arc or of similar objects may provide conclusive evidence as to which excitation mechanism applies.

Inspection of Table 1 shows that the UV lines of $\mathrm{N} V \lambda 1240$, O VI $\lambda 1035$, [Ne V] $\lambda 3426, \mathrm{Mg}$ II $\lambda \lambda 2800$ and C II] $\lambda 2326$, although weak and somewhat difficult to detect, would be good discriminants, since they are so much brighter in the filtered AGN case as compared to the stellar case. In the optical, we find that the $[\mathrm{OI}]$ and $[\mathrm{S} \mathrm{II}]$ lines are much stronger in the absorbed case while their detection would cause a problem to the stellar interpretation. In the absorbed case, the systematic larger strengths of all the low ionization lines result obviously from the strong soft X-ray component $(0.4-1.5 \mathrm{keV})$ which is responsible for the PIZ. Interestingly, the ratio [O II]/[O III] is unusually small in all models. This results essentially from 
the very high ionization parameter employed. The largest ratio value $(0.035)$ is produced by the absorbed powerlaw, though it is probably hopeless to expect detecting that faint a line.

\subsection{Sensitivity to input parameters}

If we had allowed the inner layers that filter the AGN continuum to absorb a significantly larger fraction of ionizing photons than $20 \%$, it would have resulted not only in weaker He II emission lines from the ENLR, but also in an ISED with many fewer photons left in the crucial $20-54.4 \mathrm{eV}$ range. The emerging absorbed continuum, although "harder", would mostly consist of soft X-ray photons. Such ISED produces a low ionization emission region not unlike LINERs as shown by Binette et al. (1996) (see also Halpern \& Steiner 1983).

The presence of internal dust within ENLR models can affect the calculated line ratios in four ways: depletion, additional gas heating, continuum transfer and line transfer (cf. 1993). Depletion, for instance, directly affects the gas phase abundances. Other scenarios about depletion and the degree of dust destruction than defined in Sect. 2.3 could have been adopted. However, it would mostly have been equivalent to specifying different gas phase abundances. Given the already large uncertainties in the metallicity of the Lynx arc, little can be added to the discussion at this stage. As for the absorption of the ionizing radiation and the contribution of dust ionization to the nebular heating, both were found to be relatively unimportant in the calculations reported. On the other hand, line transfer of the emergent lines through the dusty medium affected substantially the low ionization lines, despite the low dust-to-gas ratio assumed (Sect. 2.3). This is due to the rather large $U_{\text {neb }}$ adopted, which resulted in large columns of ionized gas and in a non-negligible extinction across the whole nebular structure (assumed ionization-bounded). Our transfer scheme assumes that the bulk of the observed line fluxes is dominated by clouds seen from the ionized front-end (rather than from the back). In the particular case of the absorbed powerlaw model (Col. 4 of Table 1), we find that the lowest excitation lines, those which emerge from the large PIZ at the back of the nebular structure, and which therefore must go through the largest dust columns ${ }^{2}$, are attenuated by factors of $\sim 3-4$ in the UV (e.g. Mg II and $\mathrm{C}$ II] ) and a factor $<2$ in the optical (e.g. [S II] $)^{3}$. All of the other line ratios, however, from moderate to high excitation, are not significantly attenuated by the dust. In the case of photoionization by a stellar ISED, the attenuation is negligible since the $\mathrm{PIZ}$ is nonexistent.

\subsection{Luminosity of the recombination lines}

While line ratios serve as discriminants between alternative excitation mechanisms, comparing luminosities of specific lines such as the recombination lines remains a useful exercise if

\footnotetext{
2 The $\mathrm{H}$ columns integrated from the front of the (ionizationbounded) slab up to the depth where the $\mathrm{H}$ ionized fraction has decreased to $50 \%$ and $1 \%$ are $1.0 \times 10^{22}$ and $2.2 \times 10^{23} \mathrm{~cm}^{-2}$, respectively (corresponding to $A_{\mathrm{V}}=0.13$ and 2.9 , respectively).

3 The entries in Table 1 have the attenuation already factored in.
}

only as a check on the plausibility of any proposed mechanism, especially when dealing with nebular spectra as unusual as that of the Lynx arc nebula. The (absorption corrected) fluxes, integrated over both arcs, are $3.9 \times 10^{-15}$ and $0.36 \times 10^{-15} \mathrm{erg}$ $\mathrm{s}^{-1} \mathrm{~cm}^{-2}$ for $\mathrm{Ly} \alpha$ and $\mathrm{H} \beta$, respectively (Fosbury et al. 2003). Assuming an amplification factor of 15 , due to the lens, and the "concordance" $\Lambda \mathrm{CDM}$ cosmology with $\Omega_{\Lambda}=0.7, \Omega_{M}=0.3$ and $h=0.67$ with $h=H_{0} / 100$, we derive intrinsic luminosities of $L_{\mathrm{Ly} \alpha}=2.9 \times 10^{43}$ and $L_{\mathrm{H} \beta}=2.6 \times 10^{42} \mathrm{erg} \mathrm{s}^{-1}$. Such luminosities are within reach of known $\mathrm{H}$ II galaxies or active nuclei. In effect, $L_{\mathrm{H} \beta}$ lies just below the upper $\mathrm{H} \beta$ luminosity end of H II Galaxies ${ }^{4}$ (Melnick et al. 2000), being of equivalent power to the high redshift Lyman break galaxies studied by Pettini et al. (1998). There is neither any obvious problem for an AGN origin for the ionizing radiation since the observed $L_{\mathrm{Ly} \alpha}$ is a few percents only of the spatially extended emission observed in powerful high redshift radio-galaxies (van Ojik et al. 1997). For instance, the detached extranuclear emission region in PKS B1243+036 (at position B1, van Ojik et al. 1996) exceeds by a factor 10 the $L_{\mathrm{Ly} \alpha}$ of the Lynx arc. Neither star formation nor nuclear activity can be ruled out from line luminosity considerations alone.

\section{Absorbed versus reddened QSOs}

In the case of the absorbed QSO scenario discussed in this paper, if the dense gas layers responsible for the filter were submitted to the direct powerlaw at a higher ionization parameter $U_{\text {fil }} \gtrsim 3$, they would, over very short timescales, be heated to Compton equilibrium temperatures $\left(>10^{6} \mathrm{~K}\right)$ much higher than that given by photoionization. The episode of absorbed nuclear continuum emission ${ }^{5}$ is therefore expected to occur only over a very small fraction of a QSO lifetime. This stage would be rapidly followed by a clearing of the gas within the ionizing cones. The external ENLR emission line-spectrum would then resemble that of the traditional powerlaw ENLR model. If the parent QSO was to reach a very high luminosity, it may even destroy by photoionization and comptonization (and possibly by wind mass-loading) a large part part of the opaque surrounding torus, opening the view of the central engine and of the BLR to a wider range of lines-of-sight and viewing angles. During this process of ionizing cone enlargement, the QSO may initially reveal itself as a very reddened AGN, similar to the objects reported by Gregg et al. (2002, and references therein). The last stage might consist of a torus-less QSO which would be hidden from direct view only within angles close to the equatorial plane of the accretion disk. This could provide a new explanation for the relative rarity of optically found type II QSOs even though such objects are now being found in X-ray deep field surveys (see Tran et al. 2000; Norman et al. 2002, and references therein).

\footnotetext{
${ }^{4}$ We infer a star formation rate of $S F R \sim 70 M_{\odot} \mathrm{yr}^{-1}$ assuming $\mathrm{H} \alpha / \mathrm{H} \beta=2.75$ and the Kennicutt's (1983) calibration: $S F R=$ $L_{\mathrm{H} \alpha} / 1.12 \times 10^{41} M_{\odot} \mathrm{yr}^{-1}$.

5 The issue of large covering factors in active nuclei is pertinent, given the recent discovery of many serendipitous Chandra sources characterized by absorption columns $>10^{23} \mathrm{~cm}^{-2}$ (Crawford et al. 2001, 2002; Gandhi et al. 2002).
} 


\section{Conclusions}

The absorbed powerlaw distribution is able to reproduce qualitatively the line-spectrum observed in the Lynx arc nebula. It also predicts (rest-frame) optical lines of [OI] and [S II] and UV lines of $\mathrm{Mg}$ II and C II] which are substantial stronger than in the hot stellar ISED case. Furthermore, any detection of even weak N v $\lambda 1240$, O vi $\lambda 1035$ or [Ne v] $\lambda 3426$ would clearly favor the absorbed AGN continuum interpretation.

Although this paper focuses primarily on line ratios, other aspects must also be considered. For instance, an enshrouded AGN would require the presence of a strong nuclear X-ray source and probably imply UV scattered continuum by the dust in the ENLR. Another aspect which should not be overlooked is the gas dynamics. In the particular case of the Lynx arc, the measured $F W H M$ is unusually narrow according to HN01, a characteristic more typical of H II nuclear regions than of ENLR, even though there exist cases of narrow profiles such as the detached nebulosity in Pks2152-69 which has a [O III] $F W H M$ as narrow as $160 \mathrm{~km} \mathrm{~s}^{-1}$ (Tadhunter et al. 1988). Finally, since the ionized gas in the Lynx arc has apparently been enriched up to $Z \sim 0.05 Z_{\odot}$, a self-consistent metallicity might be an issue for the hot stellar atmosphere model. In effect, the upper limit on $Z_{\star}$ needed to maintain a flux of hard photons (not quenched by the UV opacity of the metals) comparable to that of the zero-metallicity $80000 \mathrm{~K}$ model of Fig. 1 might have to be much lower than $5 \%$ solar. On the other hand, it is plausible that the stars have lower metallicity than the nebula in scenarios in which pollution of the gas by massive stellar winds or (a) supernova (ae) takes place.

Acknowledgements. The work of LB was supported by the CONACyT grant 32139-E and that of BG by the Alex Rodgers Traveling Fellowship, the Astronomical Society of Australia as well as the RSAA. RAEF is affiliated to the Space Telescopes Division of the Research and Space Science Department, the European Space Agency.

\section{References}

Anders, E., \& Grevesse, N. 1989, Geochim. Cosmochim. Acta, 53, 197

Binette, L., Wang, J. C. L., Zuo, L., \& Magris, C. G. 1993, AJ, 105, 797

Binette L., Wilson, A. S., \& Storchi-Bergmann, T. 1996, A\&A, 312, 365

Binette, L., Wilson, A. S., Raga, A., \& Storchi-Bergmann, T. 1997, A\&A, 327, 909

Crawford, C. S., Fabian, A. C., Gandhi, P., Wilman, R. J., \& Johnstone, R. M. 2001, MNRAS, 324, 427

Crawford, C. S., Gandhi, P., Fabian, A. C., et al. 2002, MNRAS, 333, 809

Ferruit, P., Binette, L., Sutherland, R. S., \& Pécontal, E. 1997, A\&A, 322, 73

Fosbury, R. A. E., Villar-Martín, M., Humphrey A., et al. 2003, ApJ, submitted

Gandhi, P., Crawford, C. S., \& Fabian, A. C. 2002, MNRAS, 337, 781

Gregg, M. D., Lacy, M., White, R. L., et al. 2002, ApJ, 564, 133

Halpern, J. P., \& Steiner, J. E. 1983, ApJ, 269, L37

Holden, B. P., Stanford, S. A., Rosati, P., et al. 2001, AJ, 122, 629 (HN01)

Hummer, D. G., \& Mihalas, D. 1970, MNRAS, 147, 339

Kennicutt, R. C. 1983, ApJ, 272, 54

Lazzati, D., \& Perna, R. 2003, MNRAS, in press

Maiolino, R., Marconi, A., Salvati, M., et al. 2001a, A\&A, 365, 28

Maiolino, R., Marconi, A., \& Oliva, E. 2001b, A\&A, 365, 37

Melnick, J., Terlevich, R., \& Terlevich, E. 2000, MNRAS, 311, 629

Norman, C., Hasinger, G., Giacconi, R., et al. 2002, ApJ, 571, 218

Pettini, M., Kellogg, M., Steidel, C. C., et al. 1998, ApJ, 508, 539

Schaerer, D. 2002, A\&A, 382, 28

Shields, G. A., \& Searle, L. 1978, ApJ, 222, 821

Tadhunter, C. N., Fosbury, R. A. E., di Serego Alighieri, S., et al. 1988, MNRAS, 235, 403

Tran, H. D., Cohen, M. H., \& Villar-Martin, M. 2000, AJ, 120, 562

van Ojik, R., Roettgering, H. J. A., Carilli, C. L., et al. 1996, A\&A, 313,25

van Ojik, R., Roettgering, H. J. A., Miley, G. K., \& Hunstead, R. W. 1997, A\&A, 317, 358

Wada, K., \& Norman, C. A. 2002, ApJ, 566, L21 